\title{
Strategies for solving the excited-state self-consistent-field problem for highly excited and multiply ionized states
}

\author{
Michael Obermeyer,,$^{1,2}$ Ludger Inhester $\oplus^{1,3, *}$ and Robin Santra $\odot^{1,2,3, \dagger}$ \\ ${ }^{1}$ Center for Free-Electron Laser Science, Deutsches Elektronen-Synchrotron DESY, Notkestrasse 85, 22607 Hamburg, Germany \\ ${ }^{2}$ Department of Physics, Universität Hamburg, Notkestrasse 9-11, 22607 Hamburg, Germany \\ ${ }^{3}$ The Hamburg Centre for Ultrafast Imaging, Luruper Chaussee 149, 22761 Hamburg, Germany
}

(Received 31 May 2021; accepted 12 August 2021; published 31 August 2021)

\begin{abstract}
The dynamics of molecules exposed to intense x-ray radiation involve a large number of multiply ionized and highly excited electronic configurations. To model these dynamics a reliable and efficient electronic structure model is imperative. Employing the Hartree-Fock-Slater electronic structure model in combination with the maximum overlap method, we quantify the associated convergence failures when calculating electronic states of carbon monoxide with multiple vacancies in the core and valence levels. We characterize these cases and describe strategies to overcome the convergence problems. The described techniques not only eliminate all convergence issues for $\mathrm{CO}$ but also result in a significant reduction of convergence failures for simulations of the $\mathrm{x}$-rayinduced multiple ionization dynamics of the phenol molecule.
\end{abstract}

DOI: 10.1103/PhysRevA.104.023115

\section{INTRODUCTION}

$\mathrm{X}$-ray free-electron lasers (XFELs) provide extremely short and ultraintense $\mathrm{x}$-ray pulses [1-6]. Their unique properties have been used to study a diverse range of phenomena [7-14]. For many XFEL experiments a detailed, microscopic understanding of the radiation damage during the pulse exposure is crucial, e.g., to estimate the structural degradation in diffractive imaging experiments $[8,15]$ or to interpret time-dependent spectroscopic signals $[16,17]$. However, the strong ionization by the $\mathrm{x}$-ray pulses poses strong challenges for theoretical modeling.

To understand the interaction of intense x-ray light with matter, one must consider that a molecule interacting with the x-ray pulse may undergo a series of photoionization, fluorescence, and Auger decay processes eventually causing the ionization of a significant fraction of the electrons. It has been demonstrated that these ionization dynamics can be modeled via rate equations for the time-dependent populations of electronic configurations assuming multiple consecutive photoionization and electronic relaxation (Auger decay and fluorescence) steps [16,18-32]. To that end the relevant cross sections and rates have to be provided. In light of the vast number of exotic electronic configurations involved, it is clear that such rates and cross sections are not available from tables in the literature. Instead, these crucial parameters must be provided from electronic structure calculations. Because of the large number of electronic configurations involved, it is critical to employ an efficient and robust electronic structure method. To that end, we seek to calculate excited electronic states by employing a mean-field approximation directly solving the self-consistent-field (SCF) equation for

\footnotetext{
*ludger.inhester@desy.de

†robin.santra@desy.de
}

excited electronic configurations [33-42]. In our previous works $[16,25,28,31,43]$, we employed the excited state orbitals obtained with the Hartree-Fock-Slater (HFS) [44] method as implemented in the XMOLECULE toolkit $[25,43]$ to obtain cross sections and rates. By solving the SCF equations for each electronic configuration, the molecular orbitals are adapted to the new electronic configuration after each photoionization, fluorescence, or Auger decay step. This orbital adaptation results in a spatial redistribution of the initially created charge in the molecule [25], which turned out to be a key mechanism for the response of molecules exposed to intense $\mathrm{x}$-ray light $[25,28]$.

In this work, we report on strategies to solve the HFSSCF equations to obtain a robust and efficient description for the many multiply ionized, excited electronic configurations. We analyze cases of convergence failure, and provide techniques to resolve them, for an exemplary molecule, the carbon monoxide molecule (CO). These techniques are implemented in the XMOLECULE toolkit. For the phenol molecule, we show that the implementations lead to a significant reduction of convergence failures in the simulation of $\mathrm{x}$-ray-induced ionization dynamics.

The paper is structured as follows: In Sec. II we give a brief summary of the employed HFS electronic structure model and provide details on its implementation [25,43]. Section III introduces a benchmark setup consisting of all the electronic configurations of the $\mathrm{CO}$ molecule that can be reached via sequentially stripping off electrons. By generalizing our calculation scheme to fractional occupation numbers we show in Sec. IV that a number of convergence failures can be overcome and, furthermore, we characterize cases that cannot be addressed via this technique. In Sec. V we present a scheme to address the remaining convergence failures. Section VI contains our results for the phenol molecule. In Sec. VII we summarize and draw final conclusions. 


\section{THE HARTREE-FOCK-SLATER SCHEME FOR EXCITED STATES}

The specific implementation of the HFS-SCF excited-state method in XMOLECULE is described in Ref. [43]. Here, we only give a brief summary.

The HFS method is based on an approximate exchange interaction that is used to solve the molecular orbitals in the mean-field potential of all electrons [44]. A set of molecular orbitals (MO) is found by seeking stationary points of the total electronic energy as a functional of the electron density $\rho(\mathbf{r})$. The electron density is

$$
\rho(\mathbf{r})=\sum_{i} n_{i}\left|\phi_{i}(\mathbf{r})\right|^{2},
$$

where $n_{i} \in\{0,1,2\}$ is the occupation number of molecular orbital $\phi_{i}(\mathbf{r})$. We expand the orbitals $\phi_{i}(\mathbf{r})$ using a set of basis functions $\chi_{\mu}(\mathbf{r})$,

$$
\phi_{i}(\mathbf{r})=\sum_{\mu} C_{\mu i} \chi_{\mu}(\mathbf{r})
$$

rendering the SCF problem to be equivalent to solving the Roothaan-Hall equation [45],

$$
\mathbf{F C}=\mathbf{S C E},
$$

which is a generalized eigenvalue problem for the expansion coefficients $C_{\mu i}$ and the diagonal matrix $\mathbf{E}$ that contains the orbital energies. In Eq. (3), $\mathbf{F}$ is the Fock matrix,

$$
F_{\mu \nu}=\int d^{3} r \chi_{\mu}^{*}(\mathbf{r})\left[-\frac{1}{2} \nabla^{2}+V_{\mathrm{eff}}(\mathbf{r})\right] \chi_{\nu}(\mathbf{r}),
$$

with the effective one-particle potential

$$
V_{\mathrm{eff}}(\mathbf{r})=-\sum_{A} \frac{Z_{A}}{\left|\mathbf{r}-\mathbf{R}_{A}\right|}+\int d^{3} r^{\prime} \frac{\rho\left(\mathbf{r}^{\prime}\right)}{\left|\mathbf{r}-\mathbf{r}^{\prime}\right|}-\frac{3}{2}\left[\frac{3}{\pi} \rho(\mathbf{r})\right]^{\frac{1}{3}},
$$

and $\mathbf{S}$ is the overlap matrix,

$$
S_{\mu \nu}=\int d^{3} r \chi_{\mu}^{*}(\mathbf{r}) \chi_{v}(\mathbf{r}) .
$$

The first term on the right-hand side of Eq. (5) describes the Coulomb interaction of an electron with the nuclei, where $Z_{A}$ is the nuclear charge of atom $A$, the second term is due to the electrostatic interaction with the electron density $\rho(\mathbf{r})$, and the last term represents the Slater exchange potential [44]. Equations (4) and (5) are expressed in atomic units, where $\hbar=e=m_{e}=1$.

For the calculations considered here, we have employed a basis set constructed from a minimal set of numerical atomic orbitals obtained with the XATOM toolkit [46] calculated for the respective atomic core-hole configurations [43].

The solution of the SCF equation is obtained by iteratively solving the generalized eigenvalue problem [Eq. (3)] and using the obtained expansion coefficients of iteration $k-1$, $\mathbf{C}_{i}^{(k-1)}$, to construct a new density, $\rho^{(k)}(\mathbf{r})$, and a new Fock matrix, $\mathbf{F}^{(k)}$, until self-consistency is fulfilled. Convergence of the resulting iterative sequence is accelerated by level shifting methods [47] and the direct-inversion-of-iterative-subspace method $[48,49]$.
When considering a general, excited electronic state, the construction of the electron density in iteration $k$ involves ambiguities as the eigenvectors $\mathbf{C}_{i}^{(k-1)}$ of the previous step have to be associated with the occupation numbers $n_{i}$. Here, one has to choose a mapping that provides the occupation numbers of the current orbitals $\phi_{i}^{(k-1)}$ with coefficients $C_{\mu i}^{(k-1)}$. For the ground configuration one can employ an ordering according to the one-particle energy, i.e., the corresponding eigenvalues of the Fock matrix $\mathbf{F}^{(k-1)}$. According to such an aufbau principle the energetically lowest orbitals are occupied resulting in the electronic ground state. For an excited electronic state this association becomes problematic, because the order of eigenvalues may change during the iterative sequence. Such a change in order can result in either convergence problems or convergence to an undesired state. This problem in the convergence of excited electronic states has been termed variational collapse [35,50,51].

To overcome this issue, several techniques have been suggested [35,41,42,52-55]. In our calculations we employ the maximum-overlap method (MOM) [35,53], where the above ambiguity is resolved by assigning an occupation number to each orbital based on orbital shape rather than orbital energy. We employ a variant of the MOM, where at each iteration the occupation numbers of the orbitals are determined by a criterion given by the overlap of the molecular orbitals with given reference orbitals $[43,53]$. Given a set of $N_{\text {orb }}$ orbitals $\left\{\tilde{\phi}_{i}\right\}$ that solve the SCF problem for an $N$-electron configuration with occupation numbers $\tilde{n}_{1}, \ldots, \tilde{n}_{N_{\text {orb }}}$, we calculate an $(N-1)$-electron configuration with occupation numbers $n_{1}=\tilde{n}_{1}, \ldots, n_{j}=\tilde{n}_{j}-1, \ldots, n_{N_{\text {orb }}}=\tilde{n}_{N_{\text {orb }}}$, where an electron has been removed from the occupied orbital $\tilde{\phi}_{j}$ by using the set of orbitals $\left\{\tilde{\phi}_{i}\right\}$ as reference orbitals. These reference orbitals also serve as initial guess orbitals for the start of the SCF iterations. To assign orbital occupation numbers in each iteration, we calculate the overlaps between the current orbitals and the reference orbitals,

$$
O_{i j}^{(k-1)}=\left\langle\phi_{i}^{(k-1)} \mid \tilde{\phi}_{j}\right\rangle,
$$

and the projections of each orbital onto the set of reference orbitals with a given occupation number,

$$
P_{i}(m)=\sum_{j}^{n_{j}=m}\left|O_{i j}^{(k-1)}\right|^{2}
$$

where $j$ runs over all reference orbitals with occupation numbers $n_{j}=m$. For constructing the density in iteration $k$ [Eq. (1)], the orbital occupation numbers $n_{i}$ are chosen such that the product

$$
s=\prod_{i}\left(P_{i}\left(n_{i}\right)\right)^{n_{i}}
$$

is maximized. Note that this optimization target is slightly different from the one used in an earlier implementation [43]; Eq. (9) represents a more natural measure of the overlap between many-electron states.

Analogously to the described ionization step, we conduct electron attachment steps by taking the orbitals obtained for an $(N-1)$-electron configuration as reference orbitals for calculating orbitals for an $N$-electron configuration, where an electron has been added to a specific orbital. 


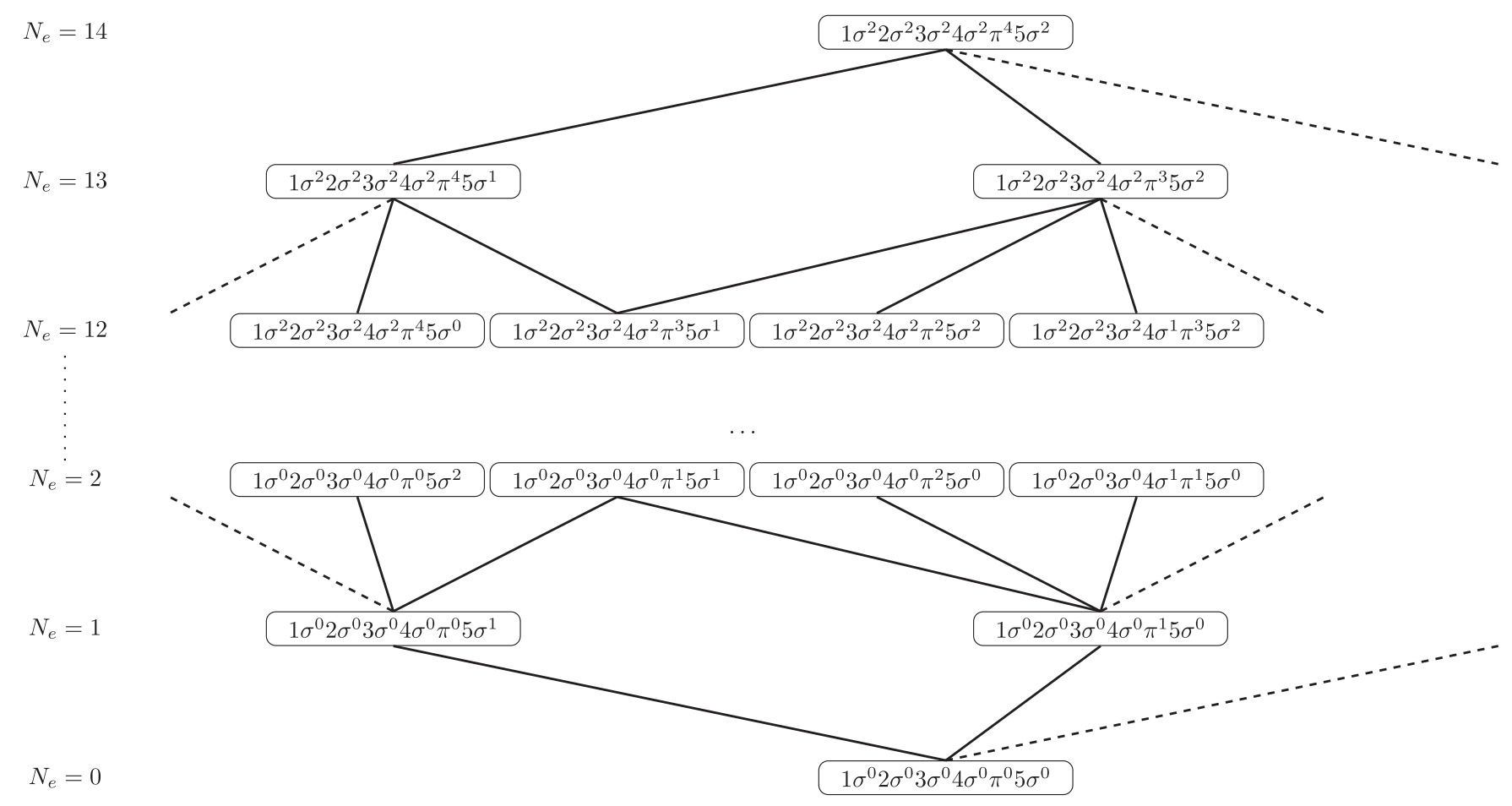

FIG. 1. Graph connecting different electronic configurations via ionization and attachment of single electrons.

\section{GRAPH OF MULTIPLY IONIZED CONFIGURATIONS}

With the methodology described in Sec. II one can calculate, in the absence of convergence issues, the full set of electronic hole configurations that occur as a result of sequential ionizations by removing one electron after another. In each ionization step, one employs the orbitals of the preceding $N$-electron configuration as reference orbitals for the target $(N-1)$-electron configuration. In a similar way, one can start with no electrons and add one electron after another into an empty or singly occupied orbital. In each such electron attachment step, one employs the orbitals of the preceding $(N-1)$-electron configuration as reference orbitals for the SCF iteration for the target $N$-electron configuration.

Such a sequential ionization or electron attachment protocol is visualized as a graph in Fig. 1 for the $\mathrm{CO}$ molecule and serves in the following as a benchmark set for the reliability of our scheme to calculate the electronic structure of generally excited and multiply ionized electronic configurations. The nodes in the graph represent electronic configurations that are ordered according to the number of electrons from bottom to top. The configurations are connected via edges that represent ionization or electron attachment processes; i.e., they connect the configurations either upwards or downwards by adding or removing an electron in a particular orbital. In total, the graph contains 1215 nodes connected via 5022 edges. Notably, in the configuration labels the orbital symbols are not necessarily ordered by energy eigenvalue. Instead, we order the orbitals by similarity with respect to shape. Thus, it may occur, for example, that the orbital $4 \sigma$ in some configurations has an orbital energy eigenvalue lower than that of the orbital $3 \sigma$. To eliminate ambiguities for the degenerate $\pi$ orbitals, we employ fractional occupation numbers that equally distribute the $\pi$ electrons between the $\pi_{x}$ and $\pi_{y}$ orbitals. The graph in Fig. 1 contains loops; i.e., one can connect two configurations via different sequences of ionization and attachment steps. For example, the electronic configuration $1 \sigma^{2} 2 \sigma^{2} 3 \sigma^{2} 4 \sigma^{2} \pi^{3} 5 \sigma^{1}$ can be reached from the neutral configuration $1 \sigma^{2} 2 \sigma^{2} 3 \sigma^{2} 4 \sigma^{2} \pi^{4} 5 \sigma^{2}$ by first stripping off an electron from the $5 \sigma$ orbital and subsequently from the $\pi$ orbital or, alternatively, by removing the electrons in opposite order.

In each ionization or electron attachment step, we quantify the orbital similarity by employing the MOM optimization target $s$ [Eq. (9)]. The quantity $s$ serves as a measure for how strongly the converged orbital sets for a given configuration overlap. An orbital set identical to the reference results in $s=1$, whereas $s=0$ if one of the occupied orbitals is orthogonal to all the occupied reference orbitals. In the following, the quantity $s$ is thus referred to as overlap between the two orbital sets.

In general, it is not guaranteed that each ionization or electron attachment step in such a graph yields a solution, since the iterative scheme described in Sec. II may not converge to a self-consistent solution within a given number of iterations. Without considering any of the implementations reported in this manuscript, we observe for the above graph for $\mathrm{CO}$ that $\sim 0.8 \%$ of the edges cannot be calculated as the SCF iterations do not converge. Moreover, we observe that for some loops the graph yields contradicting solutions for the same electronic configuration. These cases $(\sim 0.1 \%$ of the transitions involving $\sim 0.5 \%$ of the configurations) can be identified with ionization or electron attachment steps where the obtained orbitals have a particularly low overlap with the reference orbitals (small $s$ ), suggesting that the SCF iterations converged to an undesired solution. 
TABLE I. Initial and final configurations of CO for ionization steps that do not converge by smoothly adapting the occupation numbers in fractional steps.

\begin{tabular}{llc}
\hline \hline$N$-electron configuration & & $(N-1)$-electron configuration \\
\hline $1 \sigma^{1} 2 \sigma^{1} 3 \sigma^{0} 4 \sigma^{1} \pi^{1} 5 \sigma^{2}$ & $\rightarrow$ & $1 \sigma^{1} 2 \sigma^{0} 3 \sigma^{0} 4 \sigma^{1} \pi^{1} 5 \sigma^{2}$ \\
$1 \sigma^{0} 2 \sigma^{0} 3 \sigma^{0} 4 \sigma^{1} \pi^{3} 5 \sigma^{2}$ & $\rightarrow$ & $1 \sigma^{0} 2 \sigma^{0} 3 \sigma^{0} 4 \sigma^{1} \pi^{2} 5 \sigma^{2}$ \\
$1 \sigma^{1} 2 \sigma^{0} 3 \sigma^{0} 4 \sigma^{1} \pi^{2} 5 \sigma^{2}$ & $\rightarrow$ & $1 \sigma^{0} 2 \sigma^{0} 3 \sigma^{0} 4 \sigma^{1} \pi^{2} 5 \sigma^{2}$ \\
$1 \sigma^{1} 2 \sigma^{1} 3 \sigma^{0} 4 \sigma^{0} \pi^{2} 5 \sigma^{2}$ & $\rightarrow$ & $1 \sigma^{1} 2 \sigma^{0} 3 \sigma^{0} 4 \sigma^{0} \pi^{2} 5 \sigma^{2}$ \\
$1 \sigma^{1} 2 \sigma^{0} 3 \sigma^{0} 4 \sigma^{1} \pi^{1} 5 \sigma^{2}$ & $\rightarrow$ & $1 \sigma^{1} 2 \sigma^{0} 3 \sigma^{0} 4 \sigma^{1} \pi^{0} 5 \sigma^{2}$ \\
\hline \hline
\end{tabular}

\section{GENERALIZATION TO FRACTIONAL OCCUPATION NUMBERS}

Since the HFS method can also be applied to fractional occupation numbers, we propose here to traverse the graph in Fig. 1 by employing fractional occupation numbers $n_{i} \in[0,2]$. Using this generalization of the previous implementation summarized in Sec. II, we can now smoothly traverse each edge in the graph, establishing an adiabatic connection between the $N$-electron configuration and the $(N-1)$-electron configuration instead of going along an edge in a single step. To that end, the resulting orbitals of the preceding fractional ionization step are taken as reference orbitals for SCF calculation of the next fractional ionization step until the desired configuration with the integer occupation number is reached.

We make use of this scheme by refining the SCF calculation for the cases in which a single-step calculation for the ionization or attachment does not converge or yields orbitals that have low overlap with the reference orbitals. Specifically, we split an ionization or attachment step into 10 fractional steps. Employing this idea and going through all edges in the graph for $\mathrm{CO}$ where either the obtained orbital set has low overlap with the reference orbitals $(s<0.5)$ or the SCF iterations do not converge, we observe that by smoothly adapting the occupation numbers in fractional steps only $\sim 0.1 \%$ of the ionization steps still fail to converge. The ionization steps that cannot be converged are listed in Table I. Moreover, with this refined procedure, all nodes in the graph in Fig. 1 can now be reached. For a small subset of configurations $(\sim 4.3 \%$, all of them with $N_{\mathrm{el}} \leqslant 6$ ), we still obtain contradicting solutions through different ionization paths.

To investigate the remaining convergence failures, we now have a closer look at the exemplary ionization step $1 \sigma^{1} 2 \sigma^{0} 3 \sigma^{0} 4 \sigma^{1} \pi^{1} 5 \sigma^{2} \rightarrow 1 \sigma^{1} 2 \sigma^{0} 3 \sigma^{0} 4 \sigma^{1} \pi^{0} 5 \sigma^{2}$. To that end, we inspect the overlap $s$ evaluated for the configuration $1 \sigma^{1} 2 \sigma^{0} 3 \sigma^{0} 4 \sigma^{1} \pi^{0} 5 \sigma^{2}$, where the projections $P_{i}\left(n_{i}\right)$ in Eq. (9) are now constructed with orbitals that are obtained from SCF iterations employing fractional occupation numbers $f$ for the $\pi$ orbital. Because the orbitals for the target configuration are known through another path in the graph in Fig. 1, we also inspect the corresponding electron attachment process $1 \sigma^{1} 2 \sigma^{0} 3 \sigma^{0} 4 \sigma^{1} \pi^{0} 5 \sigma^{2} \rightarrow 1 \sigma^{1} 2 \sigma^{0} 3 \sigma^{0} 4 \sigma^{1} \pi^{1} 5 \sigma^{2}$. To distinguish the ionization path from the electron attachment path, we employ the additional subscripts - (for the ionization path) and + (for the electron attachment path). The violet solid line in Fig. 2 shows the overlap $s_{-}$along the ionization path as a function of the fractional occupation number

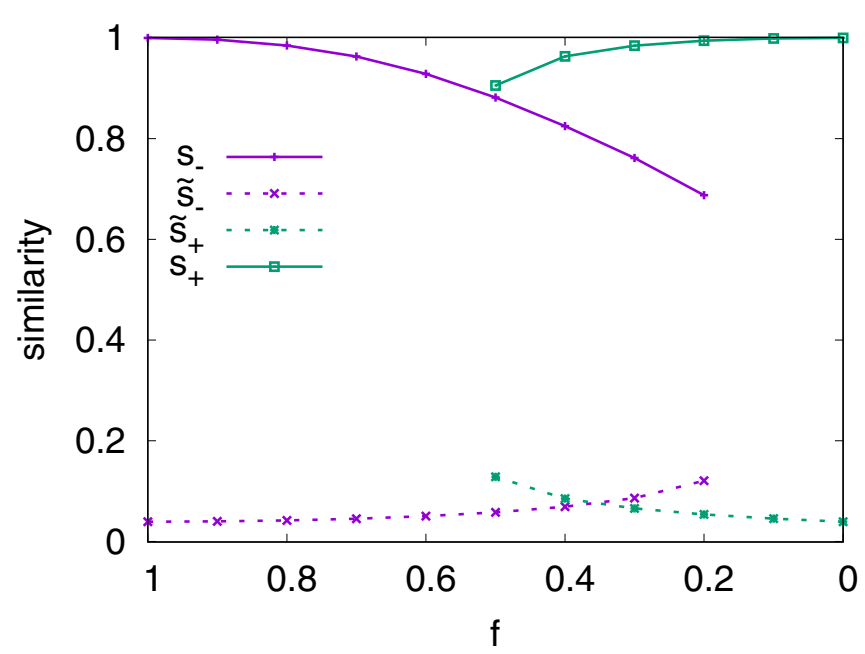

FIG. 2. Overlap $s$ between orbital sets evaluated for the configuration $1 \sigma^{1} 2 \sigma^{0} 3 \sigma^{0} 4 \sigma^{1} \pi^{f} 5 \sigma^{2}$ obtained for different values of the fractional occupation number $f$. In the missing regions no convergence is achieved. The values $s_{-}$describe the overlap of the orbital with the reference orbital set for $f=1$, and the values $\tilde{s}_{-}$describe the overlap with the reference orbitals for $f=0$ when starting with $f=1$ and successively decreasing $f$ (ionization path). The values $s_{+}$and $\tilde{s}_{+}$show the respective overlaps when starting with $f=0$ and successively increasing $f$ (electron attachment path).

$f$. As can be seen, the orbital set maintains high similarity ( $s_{-}$close to 1 ) when the fractional occupation number is smoothly reduced from 1 to 0.2 . For $f<0.2$, no convergence can be achieved. The opposite direction, the electron attachment step, is illustrated by the green solid line $\left(s_{+}\right)$starting from orbitals for the configuration $1 \sigma^{1} 2 \sigma^{0} 3 \sigma^{0} 4 \sigma^{1} \pi^{0} 5 \sigma^{2}$ and increasing $f$. Also, for the electron attachment step, the orbitals remain initially similar. For $f>0.5$ no convergence can be achieved. The dashed lines in Fig. 2 show the corresponding overlaps to the respective target orbitals $\tilde{s_{-}}$ and $\tilde{s_{+}}$. As can be seen, in the ionization as well as in the electron attachment step, the overlaps slightly increase when approaching the target configuration, but remain relatively low $\left(\tilde{s_{ \pm}}<0.2\right)$.

This result demonstrates that the solutions of the HFS equation for the two configurations cannot be connected by smoothly varying the fractional occupation number $f$. Moreover, for fractional occupation numbers $0.2 \leqslant f \leqslant 0.5$, the solution of the SCF equation depends on whether we have followed the ionization path or the electron attachment path. Qualitatively similar observations can be made for the other convergence failures listed in Table I.

Further insight into the cause of convergence failure is revealed by inspecting the total energy as a function of the fractional occupation number. For the exemplary ionization step $1 \sigma^{1} 2 \sigma^{0} 3 \sigma^{0} 4 \sigma^{1} \pi^{1} 5 \sigma^{2} \rightarrow 1 \sigma^{1} 2 \sigma^{0} 3 \sigma^{0} 4 \sigma^{1} \pi^{0} 5 \sigma^{2}$, it turns out that the convergence problems can be characterized by reducing the problem to a simplified, two-orbital model in which orbital mixing is restricted to the two-dimensional subspace spanned by the $4 \sigma$ and $5 \sigma$ orbitals of the reference configuration. These two orbitals dominate the changes between the solutions for the two configurations. 


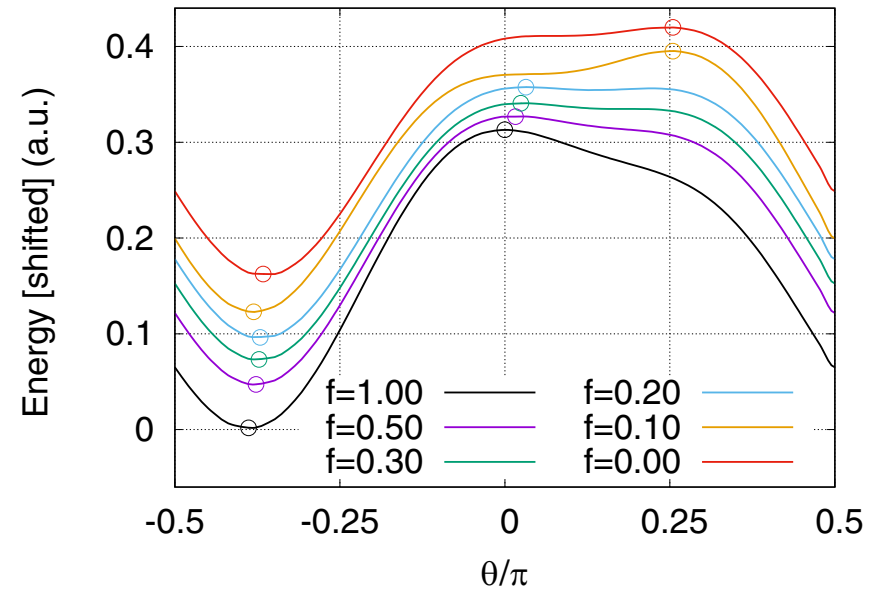

FIG. 3. HFS total energy for the configuration $1 \sigma^{1} 2 \sigma^{0} 3 \sigma^{0} 4 \sigma^{1} \pi^{f} 5 \sigma^{2}$ as a function of the rotation angle $\theta$ between the $f=1$ orbitals $4 \sigma$ and $5 \sigma$. All remaining orbitals are kept fixed at the SCF solution for the configuration with $f=1$. The reference angle $\theta \simeq 0$ corresponds to the solution for the configuration with $f=1$.

To construct such a model, we constrain the electron density $\rho(\mathbf{r})$ by employing an orbital set in which all but the $4 \sigma$ and $5 \sigma$ orbitals are kept fixed with coefficients taken from the $\mathrm{SCF}$ solution for $f=1$. Together with the orbital orthogonality constraint,

$$
\int d^{3} r \phi_{i}^{*}(\mathbf{r}) \phi_{j}(\mathbf{r})=\delta_{i j},
$$

this renders the total energy into a one-dimensional reduced energy function $[56,57]$ that depends only on the rotation angle $\theta$ between the $f=14 \sigma$ and $5 \sigma$ orbitals.

Figure 3 shows the resulting energy $E(\theta)$ for selected fractional occupation numbers. It can be seen how the stationary points of $E(\theta)$ (marked with circles) vary with the fractional occupation number $f$. The specific stationary point we are following as the solution for the configuration $1 \sigma^{1} 2 \sigma^{0} 3 \sigma^{0} 4 \sigma^{1} \pi^{f} 5 \sigma^{2}$ is the local maximum, which is for $f=1$ at $\theta=0$. The minimum at $\theta \simeq 0.375 \pi$ can be associated with the configuration $1 \sigma^{1} 2 \sigma^{0} 3 \sigma^{0} 4 \sigma^{2} \pi^{f} 5 \sigma^{1}$, where the orbitals $4 \sigma$ and $5 \sigma$ have been swapped. Notably, the angle $\theta$ between the two stationary points is not $0.5 \pi$ as one would expect from a constant mean field, i.e., where the two solutions are orthogonal to each other, indicating that the two configurations have a considerably different mean-field potential.

For fractional occupation numbers $f$ in the range from 1 to 0.3 the positions of the two stationary points shift only slightly. This illustrates that the dominant character of the SCF solutions does not change dramatically, which is in accordance with the observation that the corresponding overlap in Fig. 2 hardly changes. Accordingly, the orbitals obtained for the current fractional ionization step are similar to the SCF solutions (i.e., stationary points) of the previous fractional ionization step. The situation is, however, different when the fractional occupation number becomes lower than 0.2 . Here the maximum at $\theta \simeq 0$ vanishes, the energy $E(\theta)$ around $\theta \simeq 0$ becomes flat, and another maximum appears at $\theta \simeq 0.25 \pi$. Since the maximum suddenly moves to a different angle $\theta$, the character of the SCF solution no longer changes smoothly with the occupation number $f$, but, instead, shows a sudden change in character. The orbitals obtained from the previous fractional ionization step at $f=0.3$ are thus not appropriate reference orbitals for the SCF solution at fractional occupation number $f=0.2$. We note that such a disappearance of stationary points is reminiscent of the disappearance of SCF solutions for $\mathrm{H}_{2}$ with increasing bond length at the so-called Coulson-Fischer point [58]. Because close to the new maximum position at the angle $\theta \simeq 0.25 \pi$ both orbitals have similar overlap with both $4 \sigma$ and $5 \sigma$ reference orbitals $(\theta \simeq 0)$, it is clear that the MOM must have severe difficulties with assigning occupation numbers in each SCF iteration. By inspecting the SCF iterations, we can confirm that the restriction of the MOM enforcing high overlap with the reference orbitals hinders convergence of the SCF iterations due to repeated flipping in the assignment of occupation numbers.

The observed sudden change in character of the stationary point is a conceptual challenge for the graph in Fig. 1 that relies on the idea that SCF solutions can uniquely be identified with the removal of an electron from a specific molecular orbital. In the following, we propose a strategy to overcome the remaining convergence failures.

\section{SOLUTION FOR REMAINING CONVERGENCE FAILURES}

In Fig. 3 we have demonstrated that convergence failures, which persist even when the HFS solution is sought by means of fractional ionization steps, are linked to the sudden disappearance and appearance of stationary points in the total energy. At these points the MOM prevents the SCF iterations from reaching a stationary point since the overlap with the reference orbitals becomes too low. We therefore propose a scheme where the MOM is lifted for a selected orbital pair, thereby allowing the SCF iterations to converge to a stationary point that is not close to the provided reference orbitals. At the same time, the scheme ensures that the remaining orbitals remain similar to the provided reference orbitals.

To that end, we solve a number of constrained SCF problems, where the SCF equation is solved within a reduced subset of $n$ orbitals, while the remaining orbitals in the orthogonal $\left(N_{\text {basis }}-n\right)$-dimensional subspace are kept constant. In particular, we solve in each iteration instead of Eq. (3) the projected Roothaan-Hall equation,

$$
\left(\check{\mathbf{C}}^{\dagger} \mathbf{F} \check{\mathbf{C}}\right) \mathbf{U}=\mathbf{U} \mathbf{E}
$$

for the rotation matrix $\mathbf{U}$, where the matrix $\check{\mathbf{C}}$ has dimension $N_{\text {basis }} \times n$ and is composed of $n$ selected reference orbital coefficient vectors $\tilde{\mathbf{C}}_{i}, i=i_{1}, \ldots, i_{n}$ (the identity $\check{\mathbf{C}}^{\dagger} \mathbf{S} \check{\mathbf{C}}=\mathbf{1}$ holds). In each SCF iteration, the resulting orbital coefficients are then given by

$$
C_{\mu i}= \begin{cases}\sum_{j} \check{C}_{\mu j} U_{j k}, & \text { for } i=i_{k}, \\ \tilde{C}_{\mu i}, & \text { for } i \notin\left\{i_{1}, \ldots, i_{n}\right\} .\end{cases}
$$

Building on this, we employ the solution strategy illustrated in Fig. 4. In detail, we seek the solution through a series 


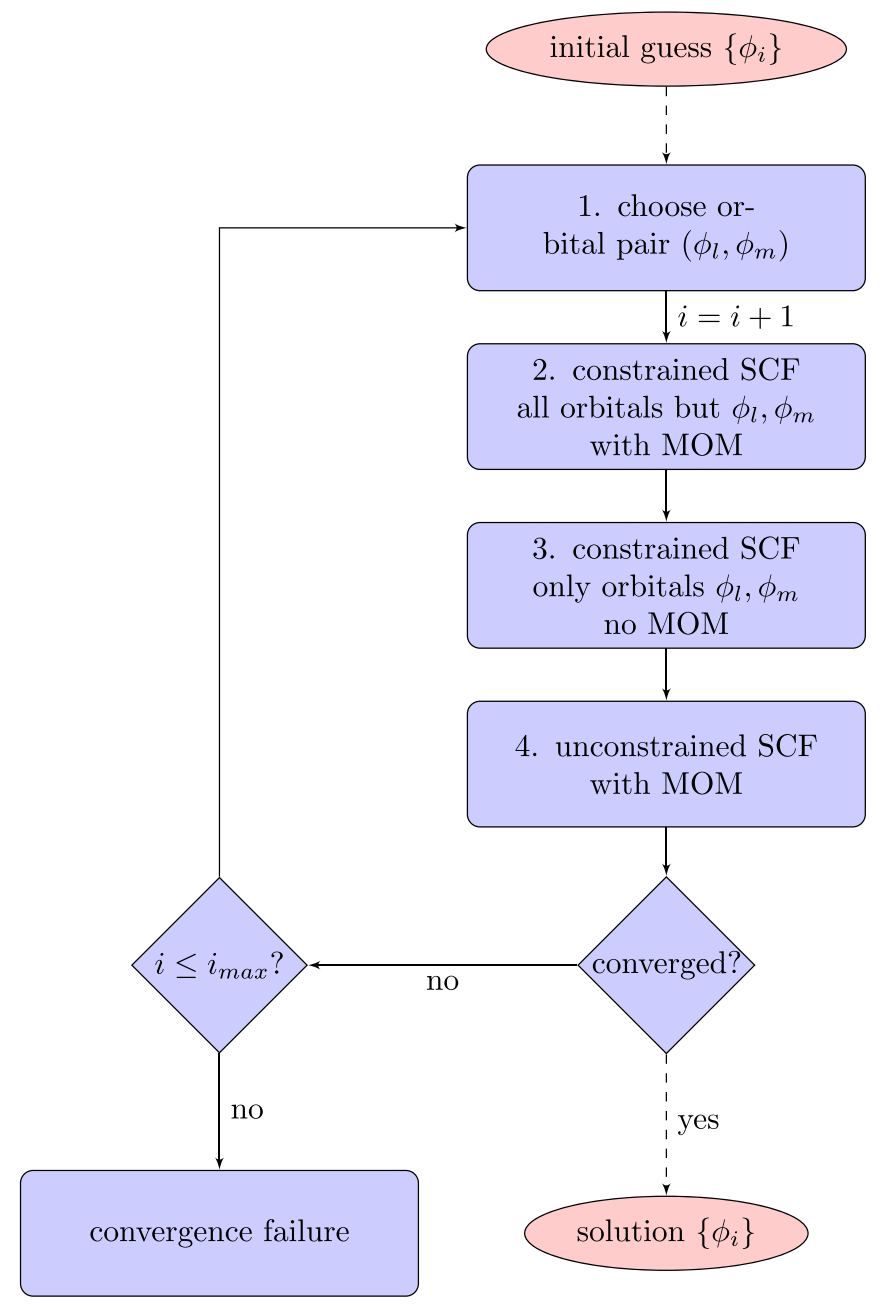

FIG. 4. Freeze-and-release strategy to resolve convergence failures.

of constrained and unconstrained SCF calculations, which we call the freeze-and-release strategy.

(i) Using the provided reference orbitals we select a pair of orbitals that shows a particularly strong change in character in the respective ionization step. This is done by calculating the Fock matrix in the MO space $\left(\mathbf{F}^{(\mathrm{MO})}=\mathbf{C}^{\dagger} \mathbf{F C}\right)$ and finding the orbital pair that has the largest nondiagonal relative Fock matrix elements, $(l, m)=\operatorname{argmax}_{(i, j)}\left|F_{i, j}^{(\mathrm{MO})} /\left(F_{i, i}^{(\mathrm{MO})}-F_{j, j}^{(\mathrm{MO})}\right)\right|$. This choice is motivated by the fact that $\mid F_{i, j}^{(\mathrm{MO})} /\left(F_{i, i}^{(\mathrm{MO})}-\right.$ $\left.F_{j, j}^{(\mathrm{MO})}\right) \mid$ is proportional to the Newton step optimizing the total energy with respect to the rotation between orbitals $i$ and $j$.

(ii) Using the reference orbitals we perform a constrained SCF calculation employing the MOM strategy in which the two orbitals $l$ and $m$ are not modified. If this calculation does not converge, we continue with the reference orbital set.

(iii) Taking the orbitals from the previous step as reference orbitals, we perform a constrained SCF calculation in which only the two orbitals $l$ and $m$ are modified; i.e., only rotations between these two selected orbitals are allowed. In this SCF calculation we do not employ the MOM, and this, therefore, allows for a more flexible solution (within the subspace of the selected orbital pair) beyond the restrictions imposed by the
MOM. Since this is effectively a one-dimensional optimization, a stationary point is usually always found.

(iv) Using the obtained orbitals from the previous step as reference orbitals, we now perform an unconstrained SCF calculation with the MOM. If this calculation does not converge, we continue with step 1 using the orbitals obtained in step 3 as a new set of reference orbitals. If the maximum number of iterations $i_{\max }=10$ of this cycle is reached, the calculation exits with convergence failure.

With the implementation of this freeze-and-release strategy, we achieve a "black-box" procedure that almost always results in a converged SCF solution and is employed if the other approaches fail. For the $\mathrm{CO}$ molecule, this procedure resolves all the remaining convergence failures listed in Table I.

\section{APPLICATION TO PHENOL}

For larger molecules, calculating the full graph as shown in Fig. 1 for the $\mathrm{CO}$ molecule or even tabulating the rates and cross sections for all electronic configurations is not possible, since the number of configurations becomes too large. To solve the rate equations for the ionization dynamics, we instead employ the Monte Carlo (MC) approach [20]; that is, we calculate configuration-space trajectories that stochastically sample the ionization dynamics of the molecule. In these simulations the electronic configurations and associated electronic transition rates are calculated on the fly. In this context, we generalize the described scheme to not only follow ionization steps but also Auger and fluorescence processes, in which two (or one) electrons are removed and another is added in the configuration. Further details on this simulation scheme are described in Refs. [28,31].

We have tested the implementations described in Secs. IV and $\mathrm{V}$ with the phenol molecule [Fig. 5(a)] exposed to an X-ray pulse with a photon energy of $2 \mathrm{keV}$, a fluence of $5 \times 10^{12}$ photons $/ \mu \mathrm{m}^{2}$, and a Gaussian temporal pulse shape with a duration of $10 \mathrm{fs}$ full width at half maximum (FWHM). Such pulse parameters are reached in the focal spot at XFELs [1]. In each electronic transition process (photoionization, fluorescence, or Auger), we try to directly converge orbitals employing the orbitals of the previous electronic configuration as reference orbitals. If in this first one-step approach the SCF iterations fail to converge or they result in a low overlap with the reference orbitals $(s<0.5)$, we calculate an SCF solution for the configuration by smoothly adapting the occupation numbers as described in Sec. IV. In case we still do not obtain a converged solution, we invoke the freezeand-release strategy described in Sec. V. If this approach still fails to yield convergence, the simulation continues with the orbital set that has been obtained for the previous electronic configuration; i.e., we continue the simulation without orbital reoptimization.

Details of the resulting charge dynamics in the phenol molecule are depicted in Figs. 5(b) and 5(c) showing the evolution of the average Mulliken charges of each atom. Our simulation indicates that the phenol molecule loses on average 35 electrons. As can be seen in Fig. 5(b), the final charge on the oxygen $(\mathrm{O})$ atom is roughly 6 , so on average the $\mathrm{O}$ atom is left behind with two electrons. The carbon (C) atoms have on average a lower final charge ranging from 3 to 4 . The lower 


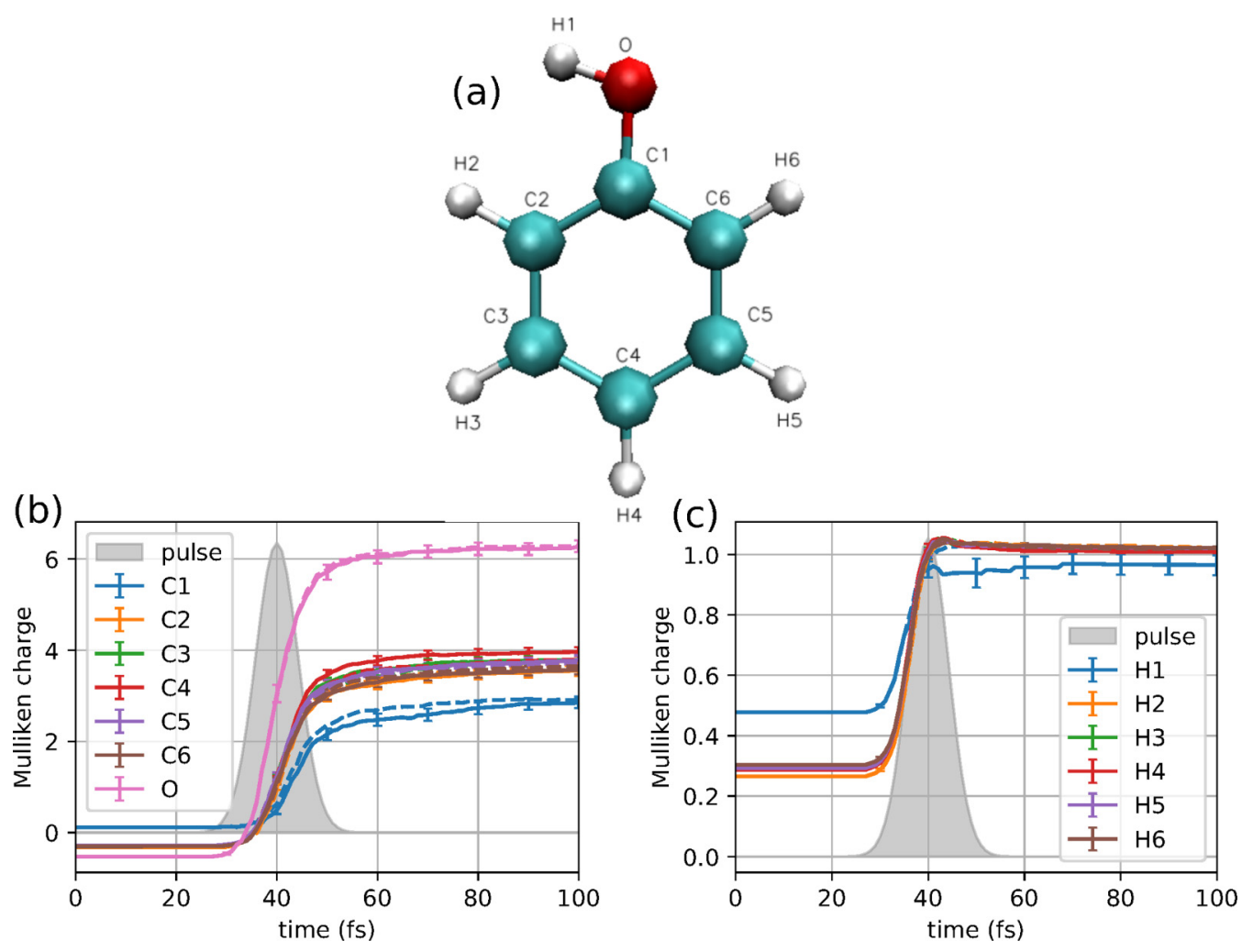

FIG. 5. Average Mulliken charges in phenol exposed to an intense soft x-ray pulse (parameters are given in the text). Panel (a) shows the geometry. Panel (b) shows the partial charges for oxygen and carbon atoms. Panel (c) shows the partial charges for hydrogen atoms. Error bars indicate standard errors due to finite sampling. The gray area indicates the x-ray pulse envelope. Dashed lines show results without invoking the freeze-and-release strategy described in Sec. V.

charge of the $\mathrm{C}$ atoms can be qualitatively understood from the $\sim 3$ times larger photoionization cross section for ionization of the $\mathrm{O} K$ shell compared to the $\mathrm{C} K$ shell at $2 \mathrm{keV}$. Among the $\mathrm{C}$ atoms, the atom $\mathrm{C} 1$, which is next to the $\mathrm{O}$ atom, stands out by having a considerably lower final charge compared to the other carbon atoms. This observation is similar to the previously reported polarization effect in iodobenzene [31], where the electrons in the benzene ring tend to be distributed so as to shield the larger charge of the neighboring heavy absorbing atom (here $\mathrm{O}$ ). The charges of the hydrogen atoms, shown in Fig. 5(c), rapidly increase and reach a value of $\simeq 1$ at the center of the pulse at $t=40 \mathrm{fs}$.

We note that the current simulations have been conducted with a fixed molecular geometry. In reality, the molecule undergoes rapid structural deformations due to the high charge in the molecule, eventually leading to a Coulomb explosion. From our previous simulations [28,31], we know that due to the high ionization rate and the short pulse duration (here 10 fs FWHM) these structural deformations have very little impact on the resulting ionization dynamics, such that the final atomic charges are barely affected. The calculated partial charges from a fixed-geometry calculation thus serves as a good approximation to the resulting ion fragment charges.

Table II lists the number of convergence failures employing the different strategies discussed in Secs. IV and V for the MC trajectories. In total we have calculated 80 trajectories that involve altogether 2824 electronic steps (photoionization, fluorescence, or Auger decay). As can be seen, without apply- ing any of the two implementations, 33\% of the electronic transition steps do not converge. The two presently implemented strategies reduce the number of convergence failures considerably. In $\sim 12 \%$ of the electronic steps, convergence could be achieved via smooth variation of occupation numbers. In almost all of the remaining cases the SCF calculations could be converged via the freeze-and-release strategy. For only $2.5 \%$ of all electronic transition steps, convergence failures remained unresolved. In these cases we continued the MC simulation with the orbitals from the previous electronic configuration.

To investigate the impact of the freeze-and-release strategy on the resulting charge dynamics, we include in Figs. 5(b) and 5(c) Mulliken charges from calculations where the freezeand-release strategy was bypassed, and we have continued the simulations with orbitals from the previous electronic step in case no convergence of the SCF calculation could be obtained through smoothly adapting the orbital occupation numbers in fractional steps. As can be seen, within the statistical fluctuation indicated by the error bars, the two simulations show the same charge evolution. These results indicate that there are no significant effects on the partial charges whether convergence is facilitated by the freeze-and-release strategy or whether the simulation continues with orbitals obtained for the preceding electron configuration. We point out, however, that it is desirable to aim for a converged solution since the apparent insensitivity to orbital reoptimization observed here for the phenol cannot be guaranteed to be a universal phenomenon. 
TABLE II. Number of SCF failures at electronic transition steps for phenol exposed to an intense $\left(5 \times 10^{12}\right.$ photons $\left./ \mu \mathrm{m}^{2}\right) \mathrm{x}$-ray pulse with a Gaussian pulse envelope (10 fs FWHM) and a photon energy of $2 \mathrm{keV}$.

\begin{tabular}{lrr}
\hline \hline & Steps & Ratio (\%) \\
\hline Total number & 2824 & 100 \\
With convergence failure & 929 & 33 \\
Cured with smooth varying of occupation number & 326 & 12 \\
Cured with freeze-and-release strategy & 533 & 19 \\
Remaining convergence failures & 70 & 2.5 \\
\hline \hline
\end{tabular}

\section{SUMMARY AND DISCUSSION}

The multiphoton multiple-ionization dynamics induced by intense $\mathrm{x}$-ray pulses involve many highly excited and ionized electronic molecular configurations that are, in general, not easy to model. The challenges lie specifically in the efficient and reliable computation of a huge number of ionized and excited electronic states. It is, therefore, imperative that the electronic structure model works as a "black box", i.e., does not involve any tuning of parameters such as selection of active orbitals or configuration spaces. To investigate these demands, we have analyzed here the graph connecting all electronic configurations that can be populated by sequentially stripping off electrons from all the orbitals of the $\mathrm{CO}$ molecule.

Within our electronic structure model, which involves a combination of HFS and MOM, we describe the implementation of techniques that yield a solution for all possible multiply ionized configurations of the $\mathrm{CO}$ molecule, employing two different strategies to overcome convergence failures. In essence, we split an ionization or electron attachment step into several fractional steps, thus making it possible to adiabatically approach the targeted SCF solution as a function of occupation number. We show that this approach does not always work, because stationary points of the total energy may disappear as a function of the occupation number. In these cases, we solve the SCF equation by partially releasing the restrictions imposed by the MOM. Applying the presented techniques to MC simulations of the ionization dynamics of phenol reveals a promising reduction of convergence failures.

Our results for the ionization dynamics in phenol illustrate that the $\mathrm{x}$-ray-induced ionization leads to an almost immediate charging of all the hydrogen atoms. The $\mathrm{O}$ atom acquires a charge considerably higher than that of the $\mathrm{C}$ atoms, and the $\mathrm{C}$ atom that connects the phenyl group with the $\mathrm{O}$ stands out as eventually having a somewhat lower charge. These results may serve as an illustrative example for the radiation damage induced by high-intensity $\mathrm{x}$-ray pulses in a biologically relevant molecule.

For the modeling of the dynamics induced by sequential X-ray multiphoton absorption, several questions remain: In most cases the response of the electronic structure to the ionization involves only a gradual change of the orbitals, suggesting a continued approximate validity of the independentparticle picture of ionization. Within the electronic mean-field picture, how to deal with ionization processes that result in an SCF solution with strongly different orbitals that cannot even be adiabatically connected remains a challenging question. For the phenol molecule we have shown that different strategies (either keeping orbitals from a previous step or following an SCF solution that has low overlap) lead to similar results for the time evolution of the charge distribution.

Further research on this is required. In particular, such questions could in the future be resolved by combining the presented SCF strategies with efficient post-SCF methods. Additional challenges arise when attempting to capture dynamical changes of the molecular geometry. The question remains as to what degree similar SCF strategies as presented here avoid convergence failures when following a continuous potential energy surface for an arbitrary excited and ionized electronic configuration.

The strategies we have demonstrated can be seen as a pragmatic recipe under the premise that the mean-field electronic structure picture may be maintained. The employed graph for CO may serve as a benchmark for the development of alternative approaches involving more refined electronic structure models.

\section{ACKNOWLEDGMENT}

This work was supported by the Chemical Sciences, Geosciences, and Biosciences Division, Office of Basic Energy Sciences, Office of Science, US Department of Energy, Grant No. DE-SC0019451.
[1] P. Emma, R. Akre, J. Arthur, R. Bionta, C. Bostedt, J. Bozek, A. Brachmann, P. Bucksbaum, R. Coffee, F.-J. Decker, Y. Ding, D. Dowell, S. Edstrom, A. Fisher, J. Frisch, S. Gilevich, J. Hastings, G. Hays, P. Hering, Z. Huang et al., Nat. Photon. 4, 641 (2010).

[2] T. Ishikawa, H. Aoyagi, T. Asaka, Y. Asano, N. Azumi, T. Bizen, H. Ego, K. Fukami, T. Fukui, Y. Furukawa, S. Goto, H. Hanaki, T. Hara, T. Hasegawa, T. Hatsui, A. Higashiya, T. Hirono, N. Hosoda, M. Ishii, T. Inagaki et al., Nat. Photon. 6, 540 (2012).
[3] E. Allaria, D. Castronovo, P. Cinquegrana, P. Craievich, M. Dal Forno, M. B. Danailov, G. D’Auria, A. Demidovich, G. De Ninno, S. Di Mitri, B. Diviacco, W. M. Fawley, M. Ferianis, E. Ferrari, L. Froehlich, G. Gaio, D. Gauthier, L. Giannessi, R. Ivanov, B. Mahieu et al., Nat. Photon. 7, 913 (2013).

[4] H.-S. Kang, C.-K. Min, H. Heo, C. Kim, H. Yang, G. Kim, I. Nam, S. Y. Baek, H.-J. Choi, G. Mun, B. R. Park, Y. J. Suh, D. C. Shin, J. Hu, J. Hong, S. Jung, S.-H. Kim, K. Kim, D. Na, S. S. Park et al., Nat. Photon. 11, 708 (2017). 
[5] W. Decking, S. Abeghyan, P. Abramian, A. Abramsky, A. Aguirre, C. Albrecht, P. Alou, M. Altarelli, P. Altmann, K. Amyan, V. Anashin, E. Apostolov, K. Appel, D. Auguste, V. Ayvazyan, S. Baark, F. Babies, N. Baboi, P. Bak, V. Balandin et al., Nat. Photon. 14, 391 (2020).

[6] E. Prat, R. Abela, M. Aiba, A. Alarcon, J. Alex, Y. Arbelo, C. Arrell, V. Arsov, C. Bacellar, C. Beard, P. Beaud, S. Bettoni, R. Biffiger, M. Bopp, H.-H. Braun, M. Calvi, A. Cassar, T. Celcer, M. Chergui, P. Chevtsov et al., Nat. Photon. 14, 748 (2020).

[7] R. Neutze and K. Moffat, Curr. Opin. Struc. Biol. 22, 651 (2012).

[8] I. Schlichting and J. Miao, Curr. Opin. Struct. Biol. 22, 613 (2012).

[9] J. C. H. Spence, U. Weierstall, and H. N. Chapman, Rep. Prog. Phys. 75, 102601 (2012).

[10] B. W. Adams, C. Buth, S. M. Cavaletto, J. Evers, Z. Harman, C. H. Keitel, A. Pálffy, A. Picón, R. Röhlsberger, Y. Rostovtsev, and K. Tamasaku, J. Mod. Opt. 60, 2 (2013).

[11] R. Alonso-Mori, K. Asa, U. Bergmann, A. S. Brewster, R. Chatterjee, J. K. Cooper, H. M. Frei, F. D. Fuller, E. Goggins, S. Gul, H. Fukuzawa, D. Iablonskyi, M. Ibrahim, T. Katayama, T. Kroll, Y. Kumagai, B. A. McClure, J. Messinger, K. Motomura, K. Nagaya et al., Faraday Discuss. 194, 621 (2016).

[12] C. Bostedt, S. Boutet, D. M. Fritz, Z. Huang, H. J. Lee, H. T. Lemke, A. Robert, W. F. Schlotter, J. J. Turner, and G. J. Williams, Rev. Mod. Phys. 88, 015007 (2016).

[13] M. Buzzi, M. Först, R. Mankowsky, and A. Cavalleri, Nat. Rev. Mater. 3, 299 (2018).

[14] L. Young, K. Ueda, M. Guehr, P. H. Bucksbaum, M. Simon, S. Mukamel, N. Rohringer, K. C. Prince, C. Masciovecchio, M. Meyer, A. Rudenko, D. Rolles, C. Bostedt, M. Fuchs, D. A. Reis, R. Santra, H. Kapteyn, M. Murnane, H. Ibrahim, F. Legare et al., J. Phys. B: At. Mol. Opt. Phys. 51, 032003 (2018).

[15] R. Neutze, R. Wouts, D. van der Spoel, E. Weckert, and J. Hajdu, Nature (London) 406, 752 (2000).

[16] J. M. Schäfer, L. Inhester, S.-K. Son, R. F. Fink, and R. Santra, Phys. Rev. A 97, 053415 (2018).

[17] X. Li, L. Inhester, T. Osipov, R. Boll, R. Coffee, J. Cryan, A. Gatton, T. Gorkhover, G. Hartman, M. Ilchen, A. Knie, M.-F. Lin, M. P. Minitti, C. Weninger, T. J. A. Wolf, S.-K. Son, R. Santra, D. Rolles, A. Rudenko, and P. Walter, Sci. Rep. 11, 505 (2021).

[18] L. Young, E. P. Kanter, B. Kraessig, Y. Li, A. M. March, S. T. Pratt, R. Santra, S. H. Southworth, N. Rohringer, L. F. DiMauro, G. Doumy, C. A. Roedig, N. Berrah, L. Fang, M. Hoener, P. H. Bucksbaum, J. P. Cryan, S. Ghimire, J. M. Glownia, D. A. Reis et al., Nature (London) 466, 56 (2010).

[19] M. Hoener, L. Fang, O. Kornilov, O. Gessner, S. T. Pratt, M. Gühr, E. P. Kanter, C. Blaga, C. Bostedt, J. D. Bozek, P. H. Bucksbaum, C. Buth, M. Chen, R. Coffee, J. Cryan, L. DiMauro, M. Glownia, E. Hosler, E. Kukk, S. R. Leone et al., Phys. Rev. Lett. 104, 253002 (2010).

[20] S.-K. Son and R. Santra, Phys. Rev. A 85, 063415 (2012).

[21] C. Buth, J. Liu, M. H. Chen, J. P. Cryan, L. Fang, J. M. Glownia, M. Hoener, R. N. Coffee, and N. Berrah, J. Chem. Phys. 136, 214310 (2012).

[22] B. Rudek, S.-K. Son, L. Foucar, S. W. Epp, B. Erk, R. Hartmann, M. Adolph, R. Andritschke, A. Aquila, N. Berrah, C. Bostedt, J. Bozek, N. Coppola, F. Filsinger, H. Gorke,
T. Gorkhover, H. Graafsma, L. Gumprecht, A. Hartmann, G. Hauser et al., Nat. Photon. 6, 858 (2012).

[23] B. Rudek, D. Rolles, S.-K. Son, L. Foucar, B. Erk, S. Epp, R. Boll, D. Anielski, C. Bostedt, S. Schorb, R. Coffee, J. Bozek, S. Trippel, T. Marchenko, M. Simon, L. Christensen, S. De, S. I. Wada, K. Ueda, I. Schlichting et al., Phys. Rev. A 87, 023413 (2013).

[24] K. Motomura, H. Fukuzawa, S.-K. Son, S. Mondal, T. Tachibana, Y. Ito, M. Kimura, K. Nagaya, T. Sakai, K. Matsunami, S. Wada, H. Hayashita, J. Kajikawa, X.-J. Liu, R. Feifel, P. Johnsson, M. Siano, E. Kukk, B. Rudek, B. Erk et al., J. Phys. B: At. Mol. Opt. Phys. 46, 164024 (2013).

[25] L. Inhester, K. Hanasaki, Y. Hao, S.-K. Son, and R. Santra, Phys. Rev. A 94, 023422 (2016).

[26] J.-C. Liu, N. Berrah, L. S. Cederbaum, J. P. Cryan, J. M. Glownia, K. J. Schafer, and C. Buth, J. Phys. B: At. Mol. Opt. Phys. 49, 075602 (2016).

[27] H. I. B. Banks, D. A. Little, J. Tennyson, and A. Emmanouilidou, Phys. Chem. Chem. Phys. 19, 19794 (2017).

[28] A. Rudenko, L. Inhester, K. Hanasaki, X. Li, S. J. Robatjazi, B. Erk, R. Boll, K. Toyota, Y. Hao, O. Vendrell, C. Bomme, E. Savelyev, B. Rudek, L. Foucar, S. H. Southworth, C. S. Lehmann, B. Kraessig, T. Marchenko, M. Simon, K. Ueda et al., Nature (London) 546, 129 (2017).

[29] K. Toyota, S.-K. Son, and R. Santra, Phys. Rev. A 95, 043412 (2017).

[30] B. Rudek, K. Toyota, L. Foucar, B. Erk, R. Boll, C. Bomme, J. Correa, S. Carron, S. Boutet, G. J. Williams, K. R. Ferguson, R. Alonso-Mori, J. E. Koglin, T. Gorkhover, M. Bucher, C. S. Lehmann, B. Krässig, S. H. Southworth, L. Young, C. Bostedt et al., Nat. Commun. 9, 4200 (2018).

[31] Y. Hao, L. Inhester, S.-K. Son, and R. Santra, Phys. Rev. A 100, 013402 (2019).

[32] S.-K. Son, R. Boll, and R. Santra, Phys. Rev. Res. 2, 023053 (2020).

[33] R. E. Stanton, J. Chem. Phys. 48, 257 (1968).

[34] K. Kowalski and K. Jankowski, Phys. Rev. Lett. 81, 1195 (1998).

[35] A. T. B. Gilbert, N. A. Besley, and P. M. W. Gill, J. Phys. Chem. A 112, 13164 (2008).

[36] N. A. Besley, A. T. B. Gilbert, and P. M. W. Gill, J. Chem. Phys. 130, 124308 (2009).

[37] A. J. W. Thom and M. Head-Gordon, Phys. Rev. Lett. 101, 193001 (2008).

[38] H. G. A. Burton and D. J. Wales, J. Chem. Theory Comput. 17, 151 (2020).

[39] R. A. Zarotiadis, H. G. A. Burton, and A. J. W. Thom, J. Chem. Theory Comput. 16, 7400 (2020).

[40] G. M. J. Barca, A. T. B. Gilbert, and P. M. W. Gill, J. Chem. Phys. 141, 111104 (2014).

[41] D. Hait and M. Head-Gordon, J. Chem. Theory Comput. 16, 1699 (2020).

[42] K. Carter-Fenk and J. M. Herbert, J. Chem. Theory Comput. 16, 5067 (2020).

[43] Y. Hao, L. Inhester, K. Hanasaki, S.-K. Son, and R. Santra, Struct. Dyn. 2, 041707 (2015).

[44] J. C. Slater, Phys. Rev. 81, 385 (1951).

[45] C. C. J. Roothaan, Rev. Mod. Phys. 23, 69 (1951). 
[46] S.-K. Son, L. Young, and R. Santra, Phys. Rev. A 83, 033402 (2011).

[47] V. R. Saunders and I. H. Hillier, Int. J. Quantum Chem. 7, 699 (1973).

[48] P. Pulay, Chem. Phys. Lett. 73, 393 (1980).

[49] P. Pulay, J. Comput. Chem. 3, 556 (1982).

[50] P. S. Bagus, Phys. Rev. 139, A619 (1965).

[51] H. J. A. Jensen, P. Jørgensen, and H. Ågren, J. Chem. Phys. 87, 451 (1987).

[52] H.-Z. Ye, M. Welborn, N. D. Ricke, and T. Van Voorhis, J. Chem. Phys. 147, 214104 (2017).
[53] G. M. J. Barca, A. T. B. Gilbert, and P. M. W. Gill, J. Chem. Theory Comput. 14, 1501 (2018).

[54] L. N. Tran, J. A. R. Shea, and E. Neuscamman, J. Chem. Theory Comput. 15, 4790 (2019).

[55] G. Levi, A. V. Ivanov, and H. Jónsson, J. Chem. Theory Comput. 16, 6968 (2020).

[56] A. Igawa and H. Fukutome, Prog. Theor. Phys. 54, 1266 (1975).

[57] Z. Tóth and P. Pulay, J. Chem. Phys. 145, 164102 (2016).

[58] P. C. A. Coulson and M. I. Fischer, London, Edinburgh, Dublin Philos. Mag. J. Sci. 40, 386 (1949). 